\title{
Applications of Microscopy in the Chemical Industry
}

\author{
Z. G. Li*, J. H. Blackson** \\ * DuPont CR\&D, Experimental Station, P. O. Box 80228, Wilmington, DE 19880-0228 \\ ** Dow Chemical, Corporate R \& D, Analytical Sciences, Bldg. 1897, Midland, MI 48667
}

The chemical industry is one of the most important and basic industrial sectors in our modern society. In fact, we use chemical-related products almost everyday. The total chemical sales of the top 50 chemical companies globally were in excess of $\$ 380$ billion in 2001 [1]. Microscopy techniques including transmission electron microscopy (TEM), scanning electron microscopy (SEM), scanned probe microscopy (SPM) and optical light microscopy (OM) have been heavily used in the chemical industry and play important roles in research and development of new and existing products. Sample preparation, as in other industries has been critical to successful microscopy based characterization of materials. Techniques employed include microtomy, metallographic polishing and focused ion beam techniques. Microscopy also plays an important role in production support as a quality control tool and in solving a variety of problems related to offspecification material. Often times, microscopy characterization is combined with other types of more traditional types of chemical analysis to formulate a total solution. We show a few recent examples to illustrate how microscopy has been important to the chemical industry.

1) TEM and more recently tapping mode AFM have proved to be indispensable tools for the characterization of polymer blends and polymer nano-composites. Structure property relationships have led to the optimization of most commercially available polymer blends. Here we discuss results on an emerging class of materials that use small additions of inorganic nanoparticles to form polymeric nanocomposites. These materials offer great promise for automotive applications where manufacturing speed, enhanced environmental and thermal stability, recyclability and weight reduction are key advantages over steel or conventional composites [2]. Efficient exfoliation of plate like materials is essential if the ultimate physical properties are to be realized. TEM and x-ray diffraction are key to determining the degree of exfoliation.

2) Faster, smaller and more efficient computer chips have resulted from shrinking the size of transistors and their interconnects. As the distance between interconnects drops to less than $100 \mathrm{~nm}$, conventional low $\mathrm{K}$ dielectric materials based on $\mathrm{SiO}_{2}$ (dielectric constant $=3.9-4.5$ ) chemistry are no longer adequate [3]. New polymer dielectric materials have been developed with a dielectric constant of 2.65 in the solid form and through the addition of air, dielectric constants of less than 2.0 have been achieved. Chemistry has been optimized to generate a low dielectric value along with high thermal stability and low moisture absorption. These materials also have the advantage that they can be spun on to the wafer rather than being deposited by CVD. Microscopy has played an important role in the development of porous low K dielectric materials and has been the "eyes" of the developers. Information such as porogen size, pore size, pore size distribution and pore location in the burned out part and the presence of large "killer pores" is readily obtained. Porous material development depends heavily on microtomy and TEM techniques and FIB-TEM techniques are used once the material is incorporated into a device. More recently X-ray scattering techniques have also been developed for on wafer characterization of porous thin films [4]. 
3) Long life Li-ion batteries have been recently utilized in cell phones and cameras. Commercial lithium-ion rechargeable batteries commonly use $\mathrm{LiCoO}_{2}$ cathode and graphite anode materials to form "rocking-chair"-type batteries capable of shuttling lithium ions back and forth by repeatedly intercalating and deintercalating lithium ions in and out of their layered structures. We have used synchrotron x-ray diffraction (SXRD) to in-situ monitor the crystalline changes in Li-ion batteries [5]. However, the microstructure of Li-ion batteries cannot be directly revealed by SXRD. We successfully obtained cross-sectional SEM and TEM images of a Li-ion battery. The cathode is composed of mesocarbon microbeads, polymer binders and other materials. It is found that each microbead is in turn made of even smaller carbon spheres and plates. Microscopy has provided needed microstructure information about the Li-ion batteries.

4) Since 1987, scientists and engineers have been developing a new generation of display devices, the organic light emitting diodes (OLED) [6]. These new devices possess many advantages over conventional display devices. They are lightweight, easy fabricated in large areas, have an unlimited choice of colors and good mechanical flexibility. Recently, several OLED products have reached the market place and several full-size commercial plants are under construction. OLED usually consist of glass substrate, indium tin oxide (ITO) anode, ultrathin hole-transport layer (HTL) and electron transport/emissive layer, and cathode. The performance of OLED strongly depends on the local microstructure such as interfaces between HTL and ITO. Microscopy was used to study in detail the microstructure of these devices. This has and will greatly impact current research and development, and future commercial production of OLEDs [7].

Through the above examples, microscopy has been shown to have broad applications in the chemical industry where it has been integral to the success of many commercially available materials. Looking forward, we believe that microscopy based techniques will play an even more important role in the chemical industry, especially in the development of new nano-structured materials/devices.

\section{References}

[1] P. L. Short, CE\&N, July 29 (2002) 16.

[2] J. M. Garces et al., Advanced Materials, 12 (2000) 1835.

[3] S. J. Martin et al., Advanced Materials, 12 (2000) 1769.

[4] B. Landes et al., International Sematech Ultra Low-K Workshop, San Francisco, California, June 6-7, 2002.

[5] Z. G. Li et al., J. Electrochemical Society (submitted).

[6] C. W. Tang, S. A. Van Slyke, Appl. Phys. Lett., 51 (1987) 913.

[7] P. E. Burrows et al., Appl. Phys. Lett., 65 (1994) 2922. 